Trends in drug-resistant tuberculosis in a gold mining workforce in South Africa,

$$
2002-2008
$$

\title{
Authors:
}

Clare L van Halsema ${ }^{1}$, Katherine L Fielding ${ }^{1}$, Violet N Chihota ${ }^{2}$, James J Lewis ${ }^{1}$, Gavin J Churchyard $^{1,2,3}$, Alison D Grant ${ }^{1}$

${ }^{1}$ London School of Hygiene and Tropical Medicine, London, UK; ${ }^{2}$ The Aurum Institute, Johannesburg, South Africa; ${ }^{3}$ Centre for AIDS Programme of Research, University of KwaZulu Natal

Running head: Drug-resistant tuberculosis among gold miners

Word count: 2500 (Summary: 200)

\section{Tables: 2}

Figures: 5

References: 26

Corresponding author: Dr Clare L van Halsema

C/o Department of Clinical Research

London School of Hygiene and Tropical Medicine

Keppel Street, London WC1E 7HT

Tel: +44(0)207927 2256

Fax: $+44(0) 2076368739$

Email: claretaylor@doctors.org.uk clare.vanhalsema@nhs.net 


\section{SUMMARY}

Setting and objective: To describe trends in drug-resistant tuberculosis in two gold mining workforces, South Africa, 2002-8.

Design: Tuberculosis programme data analysis.

Results: Tuberculosis case notification rates decreased between 2002-8, from 4006 to $3018 / 100000$ and 3192 to $2468 / 100000$ for companies A and B respectively. HIV prevalence exceeded $80 \%$ in tuberculosis episodes with known status. The proportion of tuberculosis episodes with multi-drug resistance (MDRTB) increased: among previously treated cases, from $6 / 129(4.7 \%)$ to $17 / 85(20.0 \%)$ and from $4 / 38(10.4 \%)$ to $7 / 28(25.0 \%)$ in companies $A$ and $B$ respectively (tests for trend, $p<0.001$ company $A ; p=0.304$ company $B$ ). Case notifications of MDRTB increased during 2002-8, from 39.8 to $122.9 / 100000 /$ year in company A and 57.8 to 96.8/100000/year in company B. Coverage of second-line drug susceptibility testing among MDRTB episodes was low. Previous treatment exposure was a strong risk factor for MDRTB (prevalence ratio 8.78 [95\% confidence interval $5.94-12.97$ ] in previously treated versus untreated).

Conclusion: Despite decreasing tuberculosis notifications overall, notifications of MDRTB and proportions of episodes with MDR increased in the larger company. Cure must be ensured in first episodes to prevent acquired resistance. Improved coverage of culture, drug susceptibility testing and HIV testing is required to allow treatment to be optimised.

Keywords: Multi-drug resistance, HIV, drug susceptibility testing, isoniazid, rifampicin 


\section{INTRODUCTION}

Tuberculosis case notification rates (CNR) among gold miners in South Africa are very high, exceeding 4000/100000 in 1999 [1], compared with 960/100000 nationally in South Africa in 2008 [2], attributable to high prevalence of HIV (29\% in 2001) and silicosis [3, 4]. In the 1990s, the proportion of tuberculosis episodes with multi-drug resistance was stable and relatively low in miners $[5,6]$, estimated at $1.0 \%$ and $2.8 \%$ among previously untreated and previously treated episodes respectively [5]. More recently in South Africa, transmission of drug-resistant tuberculosis and resistance to second line antituberculous agents has been reported in association with HIV treatment facilities [7] and gold miners [8].

The aims of this study were: 1) to describe trends in CNR and prevalence of drug resistance among tuberculosis episodes from 2002-8; 2) to analyse the effect of previous tuberculosis treatment and HIV infection on drug resistance in a gold mining workforce, in a period preceding a large-scale tuberculosis prevention intervention.

\section{STUDY POPULATION AND METHODS}

We analysed routine data from 2002-8 in two mining companies in Gauteng province, South Africa (companies A and B). From 2006, companies participated in "Thibela TB", a clusterrandomised trial of community-wide isoniazid preventive therapy (IPT) [9]. Of seven mine shafts contributing data for this analysis, two were intervention (starting June and July 2007) and five control clusters.

Tuberculosis episodes were included in this study if treatment started between $1^{\text {st }}$ January 2002 and $31^{\text {st }}$ December 2008. Episodes were then included in analysis of drug resistance if they were culture positive for Mycobacterium tuberculosis (without concurrent nontuberculous mycobacteria (NTM)), with drug susceptibility testing (DST) results available for at least isoniazid and rifampicin. 
Policy in both companies from 2002-8 was to perform culture and DST for rifampicin and isoniazid routinely for retreatment cases and cases responding poorly to treatment. Company A also routinely tested previously untreated cases. Second-line DST was only performed among multi-drug resistant tuberculosis (MDRTB) episodes after 2006. Medical records (electronic where available, supplemented by paper records), laboratory records and tuberculosis registers were reviewed retrospectively. For paper-based source data, for logistic reasons, complete data were only extracted for episodes with DST results.

\section{Definitions}

The CNR was defined as the number of tuberculosis treatment episodes recorded in the tuberculosis register during a given period of time, per 100000 population, with denominators calculated as below (see "data analysis").

HIV status was defined as positive if a positive rapid test or ELISA result was recorded before tuberculosis treatment was started or up to six months afterwards, and negative if a negative result was recorded after the start of tuberculosis treatment or up to six months before.

Drug resistance in previously untreated versus previously treated cases was defined as drug-resistant tuberculosis occurring in an individual with fewer than 30 versus 30 or more days previous tuberculosis treatment exposure. Changes in drug susceptibility during treatment were also counted as episodes of drug resistance in previously treated cases and included in calculating CNR for drug-resistant tuberculosis and prevalence ratios for drug resistance by treatment history and HIV status.

MDRTB was defined as $M$. tuberculosis resistant to at least rifampicin and isoniazid. Rifampicin and isoniazid monoresistance were defined as M. tuberculosis resistant to rifampicin but not isoniazid and isoniazid but not rifampicin, respectively. 


\section{Data analysis}

In order to avoid counting re-registrations of the same tuberculosis disease episode, which can occur when treatment is interrupted and then restarted, we excluded re-registrations for the same individual within six months after the planned end of treatment, unless there was a change in drug susceptibility between registrations, for calculations of CNR for and proportions with resistance. In calculating DST coverage (company A), episodes with mixed M. tuberculosis and NTM isolated were excluded from numerator and denominator to allow for difficulties in interpreting DST. Episodes included in the calculation of CNR, but excluded from estimates of episodes with DST, comprised those excluded for re-registrations; cultures not done; culture negative; no DST; cultures positive for NTM only or mixed M. tuberculosis/ NTM. Prevalence ratios were analysed using combined data from both companies. For calculating proportions with drug resistance, denominators comprised episodes with DST available. Based on existing literature [10] we anticipated that previous treatment would be the main risk factor for drug resistance. We also investigated the role of HIV infection. Chisquared tests were used to compare proportions and the score test for trend to assess for linear trend. The Poisson model was used to assess for linear trend and departures from linear trend in CNR by year. Where there was evidence for departure from linear trend the $p$ value for the overall test is quoted.

Company workforce data (mean workforce size each calendar year) were used to estimate denominators for CNR. Company A provided monthly figures from September 2003; company B provided 1-8 figures annually from September 2003. September 2003 denominators were used for earlier estimates. Permanent employees and short term contractors were included in denominators, since both contributed to numerators.

Data were entered using EpiData (www.epidata.dk) and analysed using STATA v10 (STATA Corporation, Texas). 


\section{Laboratory methods}

From 2002 laboratories serving mine health services performed culture and DST using BACTEC $^{\text {TM }}$ Mycobacterial Growth Indicator Tube 960 system [BD Diagnostics System, Sparks MD].

\section{Ethical approval}

Research Ethics Committees of the London School of Hygiene and Tropical Medicine and the University of KwaZulu Natal approved the study.

\section{RESULTS}

\section{Study population}

Workforce size, 2002-8, ranged from 15239 to 19476 (company A) and from 6671 to 8414 (company B). Median age was 42 years (interquartile range [IQR] 37, 47 years) for company A and 44 years (IQR 40, 48) for company B.

\section{Numbers included in analysis and case notification rates (CNR)}

In company A, with 4268 tuberculosis episodes registered from 2002-8, CNR decreased from 4006/100000 in 2002 to $3018 / 100000$ in 2008 (overall $p<0.001$ ). Of 4268 episodes registered, exclusions from the analyses for this study were: 1807 not culture positive; 78 reregistrations; 133 mixed $M$. tuberculosis/NTM; 243 lacked DST for isoniazid and/or rifampicin. Therefore 2007/4268 (47.0\%) registered, culture positive M. tuberculosis episodes with DST for rifampicin and isoniazid were included in analyses for company A (figure 1). Of these, $617 / 2007(30.7 \%)$ were retreatment episodes. Five episodes with change in drug susceptibility during treatment were also included. 
For company B, 1472 episodes were registered from 2002-8 and CNR decreased from $3192 / 100000$ in 2002 to $2468 / 100000$ in 2008 (test for trend, $p<0.001$ ). Of 1472 episodes registered, exclusions from the analyses for this study were: 1014 not culture positive or culture positive with DST not available for rifampicin and/or isoniazid; 43 re-registrations. Therefore 415/1472 (28.2\%) registered, culture positive M. tuberculosis episodes with DST for rifampicin and isoniazid were included in the below analyses for company B (figure 1). Of these, 200/415 (48.2\%) were retreatment episodes. Four episodes with change in drug susceptibility during treatment were also included.

CNR and numbers of culture-positive $M$. tuberculosis episodes with DST for rifampicin and isoniazid after all exclusions are shown in figure 2.

\section{Coverage of drug susceptibility testing}

For company A, first-line DST (at least rifampicin and isoniazid) was done in 2007/2250 $(89.2 \%)$ of $M$. tuberculosis culture-positive episodes. For episodes registered as retreatment episodes, DST coverage was $617 / 691(89.3 \%)$ in total and $82 / 82(100 \%)$ in 2008 . Coverage was not calculable for company B as complete data were only collected on episodes with DST available.

\section{HIV prevalence}

In company A, from 2002-8, HIV status was documented for 1329/2012 (66.1\%) episodes with DST available. Of these, 1160 (87.3\%) were HIV-infected. The prevalence of HIV infection increased from 149/173 (86.1\%) in 2002 to 153/167 (91.6\%) in 2008 (test for trend $\mathrm{p}=0.086)$.

In company B, from 2002-8, HIV status was documented for 157/419 (37.5\%) episodes with DST available. Of these, 135 (86.0\%) were HIV-infected. 
Trends in drug-resistant tuberculosis: case notification rates (previously treated and untreated cases combined)

In company A, MDRTB CNR increased from 39.8/100000/year in 2002 to 122.9/100000/year in 2008 (test for trend $p=0.004$ ) and CNR for isoniazid monoresistant episodes changed by year (overall $p<0.001$ ) without linear trend. CNR for rifampicin monoresistant episodes was 17.1/100000 in 2002 and 55.3/100000 in 2008. In company B, MDRTB CNR increased from 57.8/100000/year in 2002 to $96.8 / 100000 /$ year in 2008 and CNR for isoniazid monoresistant episodes was 72.2/100000 in 2002 and 72.6/100000 in 2008, neither showing evidence of linear increase (tests for trend, $\mathrm{p}=0.65$ and $\mathrm{p}=0.73$ respectively) (figure 3 ).

\section{Trends in drug resistance among previously untreated cases}

In company $A$, the proportion of previously untreated cases with MDRTB rose from 2/245 $(0.8 \%)$ in 2002 to $3 / 125(2.4 \%)$ in 2008 (test for trend, $p=0.005)$. In company $B$, few MDRTB episodes were registered among previously untreated cases (figure 4).

\section{Trends in drug resistance among previously treated cases}

In company A, MDRTB occurred in 6/129 (4.7\%) of episodes with previous treatment exposure in 2002 and 17/85 (20.0\%) in 2008 (test for trend $p<0.001$ ). Rifampicin monoresistance occurred in 2/129 (1.6\%) in 2002 and 7/85 (8.2\%) in 2008 (figure 5).

In company B only two episodes were rifampicin monoresistant. MDRTB occurred in 4/38 episodes (10.4\%) in 2002 and 7/28 (25.0\%) in 2008 (test for trend, $\mathrm{p}=0.304)$ (figure 5). 


\section{Second-line DST}

From $2002-8$, in company A, 88/124 (71.0\%) of MDRTB cases were tested for ethambutol and streptomycin susceptibility and 48/88 (54.5\%) were resistant to both drugs. In company B, 11/44 (25.0\%) of MDRTB cases were tested: $8 / 11$ were streptomycin resistant, with $3 / 8$ also ethambutol resistant.

For company A, 7/69 MDRTB episodes in 2006-8 had second line DST results available. In company B, second line DST was documented in 1/20 MDRTB episodes in 2006-8.

\section{Drug resistance by previous treatment exposure and HIV status}

Table 1 shows the proportions with drug resistance among previously treated versus untreated episodes. Previous treatment was a strong risk factor for drug resistance, with prevalence ratio for MDRTB of 8.78 (95\% confidence interval [CI] $5.94-12.97)$ and for rifampicin monoresistance of $6.11(95 \% \mathrm{Cl} 2.60-14.37)$ in previously treated versus untreated cases. Previous treatment exposure was less strongly associated with isoniazid monoresistance (prevalence ratio 1.45 [95\% Cl $1.15-1.83]$ ). Results were very similar when stratified by company (data not shown).

Among previously untreated episodes, there was no evidence of association between HIV infection and MDRTB, with prevalence ratio for HIV-infected versus HIV-uninfected of 0.95 $(95 \%$ Cl $0.28-3.15)$ (table 2). Among previously treated cases, 87/500 (17.4\%) episodes were MDRTB among HIV co-infected, versus 8/72 (11.1\%) among HIV uninfected (prevalence ratio 1.57 [95\% Cl $0.79-3.09]$ ). There was no evidence for interaction between HIV status and previous treatment exposure $(\mathrm{p}=0.202)$.

Among previously treated cases, HIV infection was not associated with rifampicin resistance. 102/500 (20.4\%) episodes were rifampicin resistant (monoresistant or MDRTB) among HIV 
infected, versus 9/72 (12.5\%) among HIV uninfected (prevalence ratio 1.63 [95\% Cl $0.86-$ 3.08]) (table 2).

\section{DISCUSSION}

Although CNR among miners have fallen since 2002, they remain very high. Reasons for the observed decrease are not clear, but may include higher antiretroviral therapy coverage, which we will investigate in future work. We do not know of any factors that may have caused completeness of notifications to change over the study period. High CNR may partly be attributed to case detection through annual workforce surveillance by chest radiograph. Comparisons with South African national rates must consider the denominator population of a workforce with strong tuberculosis risk factors.

Despite CNR decreasing overall, MDRTB CNR in company A increased. Although several factors may be contributory, including artefact due to higher DST coverage, we consider this to be unlikely to account for all the increase in MDRTB CNR seen here; which is more likely to be a true increase as rifampicin resistance is superimposed on existing isoniazid resistance. Isoniazid monoresistance CNR changed by year in company $A$, but without evidence of linear increase in either company. Previous studies suggest that rifampicin levels are reduced among HIV-infected individuals [11] and that individual pharmacokinetic variability accounts for some rifampicin resistance [12]. Drugs used in mine health services are obtained from approved national sources and rifampicin under-dosing is unlikely. We do not have data on treatment adherence in this population but are not aware of any factors that may have caused it to change with time.

Proportions with rifampicin- and multi-drug resistance are higher than reported previously in similar populations $[5,6]$. Such resistance was associated with previous treatment exposure, consistent with acquisition of resistance due to previous inadequate treatment, as seen in a 
similar workforce [13]. However, two studies using genotyping, one in a mining population, suggest that circulating MDR strains are also transmitted among previously treated individuals $[8,14]$ and this will account for a proportion of the resistance seen here.

HIV infection was not a strong risk factor for drug resistance. A South African national survey in 2001-2 suggested some association between HIV infection and MDRTB in previously treated cases [15]. Similarly for rifampicin monoresistance, these results do not exclude an effect of HIV infection among previously treated cases, but numbers are insufficient to conclude that observations are not due to chance. International data, collated in a systematic review, were also inconclusive on this point [16].

There is inherent uncertainty regarding population CNRs of drug-resistant disease in this observational study as numerators are relatively small. Bias cannot be excluded due to suboptimal DST coverage. DST may have been more likely to be performed when resistance was suspected, resulting in overestimation of observed proportions, where DST was not done systematically. It is unlikely that changes in proportions with MDRTB are entirely due to changes in testing coverage as our data show increased DST coverage over the study period, which may reflect less selective, more routine testing, or possibly an increase in proportions with suspected drug resistance.

HIV status was not documented for all episodes notified, but HIV prevalence among episodes with known status was very high throughout the study period. Strategies for improved HIV testing and treatment coverage are essential to reduce risk of tuberculosis [17], particularly in view of recent evidence $[18,19,20]$ and revised World Health Organization recommendations for early antiretroviral therapy for all HIV-infected individuals with active tuberculosis [21]. This is particularly important for those with drug-resistant tuberculosis and HIV, among whom mortality is high [22, 23, 24]. 
This study was conducted in a period preceding widespread IPT. There has been concern that IPT may lead to increased isoniazid resistance, although there is no evidence for this $[25,26]$. Considering that the intervention began in parts of this workforce from June 2007, the period of this analysis is likely to be too early to reflect its impact. Furthermore, IPT is unlikely to have resulted in the increase in MDRTB seen here and there was no evidence of increasing isoniazid monoresistance.

\section{CONCLUSIONS}

Despite decreasing tuberculosis CNR, MDRTB CNR and proportions of episodes with drug resistance increased in the larger company. It is essential to ensure cure of first episodes to prevent acquired resistance and to ensure prompt recognition, treatment and cure of drugresistant tuberculosis to prevent further resistance and transmission. Tuberculosis control in this high risk population is challenging and improved coverage of culture, DST and HIV testing is essential to ensure appropriate treatment for tuberculosis. 


\section{ACKNOWLEDGEMENTS}

\section{Financial Support:}

This project was funded by a grant from the Colt Foundation, UK, with additional support from Thibela TB (funded by the Consortium to Respond Effectively to the AIDS/TB Epidemics (CREATE), through a grant from the Bill and Melinda Gates Foundation) and the U.S. National Institutes of Health (grant number AI077486 to Susan E. Dorman).

CvH was supported by a grant from the Colt Foundation, UK

KF and JL are supported by the Biostatistics Core of CREATE. KF was also supported by the U.S. National Institutes of Health.

VC was supported by the NIH Fogarty ICORTA-TB/AIDS grants 5U2RTW007370 and 5U2RTW007373

AG was supported by a public health career scientist award from the Department of Health, UK

\section{Author contributions}

CLvH: Study design and implementation, data analysis, paper writing

KLF: Study concept and design, statistical and epidemiological input, data analysis, manuscript review

VNC: Study implementation and design, manuscript review

JJL: Statistical advice, data analysis, manuscript review

GJC: Study design, context advice, manuscript review

ADG: Study concept and design, epidemiological advice, paper writing 
Other contributions to the study:

Authors are grateful to the staff of Thibela TB.

We thank mining companies for permission to publish these data and the staff of gold mine health services for their assistance in facilitating data collection. 


\section{REFERENCES}

1. Corbett EL, Churchyard GJ, Charalambous S, et al. Morbidity and mortality in South African gold miners; impact of untreated disease due to human immunodeficiency virus. Clin Infect Dis 2002;32(9):1251-1258

2. World Health Organization. Global Tuberculosis Control: WHO Report 2010. World Health Organization, Geneva 2010. (WHO/HTM/TB/2010.07)

3. Lewis JJ, Charalambous S, Day JH, et al. HIV infection does not affect active case finding of tuberculosis in South African gold miners. Am J Respir Crit Care Med $2009 ; 180(12): 1271-1278$

4. Corbett EL, Churchyard GJ, Clayton TC, et al. HIV infection and silicosis: the impact of two potent risk factors on the incidence of mycobacterial disease in South African miners. AIDS 2000; 14(17): 2759-2768

5. Churchyard GJ, Corbett EL, Kleinschmidt I, Mulder D, De Cock KM. Drug-resistant tuberculosis in South African gold miners: incidence and associated factors. Int $\mathrm{J}$ Tuberc Lung Dis 2000;4(5):433-440

6. Murray J, Sonnenberg P, Shearer S, Godfrey-Faussett P. Drug-resistant pulmonary tuberculosis in a cohort of southern African goldminers with a high prevalence of HIV infection. S Afr Med J 2000;90(4):381-386

7. Gandhi NR, Moll A, Sturm AW, et al. Extensively drug-resistant tuberculosis as a cause of death in patients co-infected with tuberculosis and HIV in a rural area of South Africa. Lancet 2006;368(9547):1575-1580

8. Calver AD, Falmer AA, Murray M, et al. Emergence of increased resistance and extensively drug-resistant tuberculosis despite treatment adherence, South Africa. Emerg Infect Dis 2010;16(2):264-271 
9. Fielding, KL, Grant AD, Hayes RJ, Chaisson RE, Corbett EL, Churchyard GJ. Thibela TB: Design and methods of a cluster randomised trial of the effect of community-wide isoniazid preventive therapy on tuberculosis among gold miners in South Africa. Contemp Clin Trials 2011;32(3):382-392

10. World Health Organization 2008. Anti-tuberculosis drug resistance in the world: fourth global report. World Health Organization, Geneva, 2008. WHO/HTM/TB/2008.394

11. Chudeya S, Winston CA, Peloquin CA, et al. Isoniazid, rifampicin, ethambutol and pyrazinamide pharmacokinetics and treatment outcomes among a predominantly HIV-infected cohort of adults with tuberculosis from Botswana. Clin Infect Dis $2009 ; 48: 1685-1694$

12. Srivastava S, Pasipanodya JG, Meek C, Leff R, Gumbo T. Multidrug-resistant tuberculosis not due to noncompliance but to between-patient pharmacokinetic variability. J Infect Dis 2011;204 (12);1951-1959

13. Godfrey-Faussett P, Sonnenberg P, Shearer SC, et al. Tuberculosis control and molecular epidemiology in a South African gold-mining community. Lancet 2000;356(9235):1066-1071

14. Van Rie A, Warren R, Richardson M, et al. Classification of drug-resistant tuberculosis in an epidemic area. Lancet 2000;356(9223):22-25

15. Weyer K, Lancaster J, Brand J, van der Walt M, Levin J. Survey of Tuberculosis Drug Resistance in South Africa 2001-2002. MRC South Africa 2004. Available at http://www.sahealthinfo.org/tb/tbsurvey.htm . Accessed 7th February 2011. 
16. Suchindran S, Brouwer ES, Van Rie A. Is HIV infection a risk factor for multi-drug resistant tuberculosis? A systematic review. PLoS One 2009;4(5):e5561 doi 10.1371/journal.pone.0005561

17. Lawn SD, Myer L, Edwards D, Bekker LG, Wood R. Short-term and long-term risk of tuberculosis associated with CD4 cell recovery during antiretroviral therapy in South Africa. AIDS 2009;23(13):1717-1725

18. Abdool Karim SS, Naidoo K, Grobler A, et al. Timing of initiation of antiretroviral drugs during tuberculosis therapy. N Engl J Med 2010;362(8):697-706

19. Blanc FX, Sok T, Laureillard D, et al. Earlier versus later start of antiretroviral therapy in HIV-infected adults with tuberculosis. N Eng J Med 2011;365(16):1471-1481

20. Havlir DV, Kendall MA, Ive P, et al. Timing of antiretroviral therapy for HIV-1 infection and tuberculosis. N Eng J Med 2011;365(16):1482-1491

21. World Health Organization. Antiretroviral therapy for HIV infection in adults and adolescents: recommendations for a public health approach - 2010 revision. World Health Organization, Geneva 2010.

22. Seung KJ, Omatayo DB, Keshavjee S, Furin JJ, Farmer PE, Satti H. Early outcomes of MDR-TB treatment in a high HIV-prevalence setting in southern Africa. PLoS One 2009;4(9):e7186. Doi:10.1371/journal.pone.0007186

23. Hassim S, Shaw PA, Sangweni $P$, et al. Detection of a substantial rate of multidrugresistant tuberculosis in an HIV-infected population in South Africa by active monitoring of sputum samples. Clin Infect Dis 2010;50:1053-1059

24. Gandhi NR, Shah NS, Andrews JR, et al. HIV coinfection in multidrug- and extensively drug-resistant tuberculosis results in high early mortality. Am J Resp Crit Care Med 2009;181:80-86 
25. van Halsema CL, Fielding KL, Chihota VN, et al. Tuberculosis outcomes and drug susceptibility in individuals exposed to isoniazid preventive therapy in a high HIV prevalence setting. AIDS 2010;24: 1051-5

26. Balcells ME, Thomas SL, Godfrey-Faussett P, Grant AD. Isoniazid preventive therapy and risk for resistant tuberculosis. Emerg Infect Dis 2006;12:744-51 


\section{TABLES AND FIGURES}

Table 1: Association of antituberculous drug susceptibility patterns with treatment history, 2002 to 2008: Data from companies A and B combined.

\begin{tabular}{|c|c|c|c|}
\hline $\begin{array}{l}\text { Drug susceptibility } \\
\text { pattern }\end{array}$ & $\begin{array}{c}\text { Previously } \\
\text { untreated episodes } \\
\text { n=1547 } \\
n(\%)\end{array}$ & $\begin{array}{c}\text { Previously treated } \\
\text { episodes } \\
n=884^{2} \\
n(\%)\end{array}$ & $\begin{array}{l}\text { Prevalence ratio } \\
(95 \% \text { confidence } \\
\text { interval })^{\star}\end{array}$ \\
\hline $\begin{array}{l}\text { Rifampicin and isoniazid } \\
\text { susceptible }\end{array}$ & $1359(87.8)$ & $619(70.0)$ & 1.0 (reference) \\
\hline $\begin{array}{l}\text { Isoniazid resistant, } \\
\text { rifampicin susceptible }\end{array}$ & $152(9.8)$ & $106(12.0)$ & $1.45(1.15-1.83)$ \\
\hline $\begin{array}{l}\text { Rifampicin resistant, } \\
\text { isoniazid susceptible }\end{array}$ & $7(0.5)$ & $20(2.3)$ & $6.11(2.60-14.37)$ \\
\hline MDR & $29(1.9)$ & $139(15.7)$ & $8.78(5.94-12.97)$ \\
\hline Isoniazid any resistance & $181(11.7)$ & $245(27.7)$ & $2.41(2.03-2.87)$ \\
\hline \multicolumn{4}{|c|}{$\begin{array}{l}\text { Prevalence ratios calculated separately for each drug susceptibility pattern compared with rifampicin } \\
\text { and isoniazid susceptible episodes, ratio compares those previously treated with those previously } \\
\text { untreated } \\
{ }^{2} \text { Total includes all retreatment episodes, plus } 9 \text { changes in DST during treatment of first tuberculosis } \\
\text { episodes and } 58 \text { first treatment episodes for which DST was done on specimens taken after } 30 \text { or } \\
\text { more days' treatment exposure }\end{array}$} \\
\hline
\end{tabular}


Table 2: Multi-drug resistance, rifampicin and isoniazid resistance among tuberculosis episodes in HIV-infected and -uninfected individuals in 2 companies $\mathrm{A}$ and $\mathrm{B}$ combined

\begin{tabular}{lcccc}
\hline Number of episodes & $\begin{array}{c}\text { HIV infected: } \\
\text { resistant/total }{ }^{1}(\%)\end{array}$ & $\begin{array}{c}\text { HIV uninfected: } \\
\text { resistant/total }{ }^{1}(\%)\end{array}$ & $\begin{array}{c}\text { Prevalence ratio } \\
{ }^{2}\end{array}$ & $\begin{array}{c}\mathrm{p} \text {-value } \\
\text { (95\% confidence interval) }\end{array}$ \\
\hline MDR & $19 / 795(2.4)$ & $3 / 119(2.5)$ & $0.95(0.28-3.15)$ & 0.931 \\
\hline Previously untreated & $87 / 500(17.4 \%)$ & $8 / 72(11.1)$ & $1.57(0.79-3.09)$ & 0.180 \\
\hline Previously treated & & & \\
\hline
\end{tabular}

Any rifampicin resistance (MDR plus rifampicin monoresistance)

\begin{tabular}{|c|c|c|c|c|}
\hline Previously untreated & $21 / 795(2.6)$ & $5 / 119(4.2)$ & $0.63(0.24-1.64)$ & 0.340 \\
\hline
\end{tabular}

\section{Any isoniazid resistance (MDR plus isoniazid monoresistance)}

\begin{tabular}{lccc}
\hline Previously untreated & $108 / 795(13.6)$ & $12 / 119(10.1)$ & $1.35(0.77-2.37)$ \\
\hline Previously treated & $144 / 500(28.8)$ & $18 / 72(25.0)$ & $1.15(0.75-1.76)$ \\
\hline
\end{tabular}

37 Denominators include all registered episodes with DST available and known HIV status $(n=1480)$ plus six cases of change of drug susceptibility during 4 treatment in individuals with known HIV status (total=1486)

$5 \quad{ }^{2}$ Prevalence of resistance pattern specified, in HIV infected versus uninfected 
$6 \quad$ Figure 1: Flow chart of numbers of tuberculosis episodes included at each stage of results

7 and analyses

Total number of episodes registered 2002-8 (CNR)

Episodes culture positive for $M$. tuberculosis

Culture positive episodes with DST available for rifampicin and isoniazid

Re-registrations excluded

Corrected number after excluding re-registrations

Excluded from DST coverage calculations as M. tuberculosis and NTM isolated in culture

Culture positive episodes with $M$. tuberculosis only isolated

Registered culture positive $M$. tuberculosis with DST available Including change of DST during treatment of a registered episode

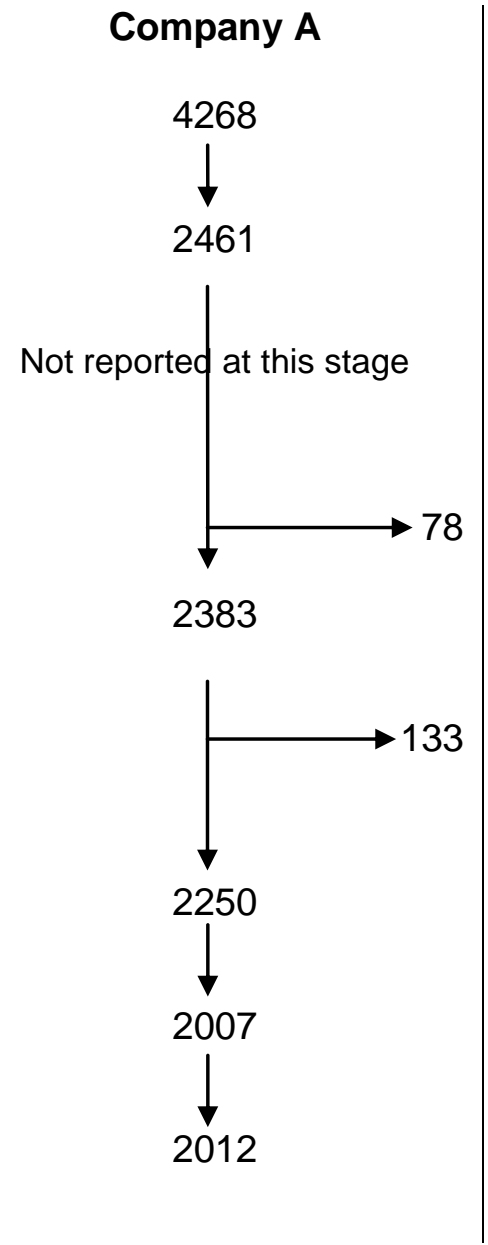

\section{Company B}

1472

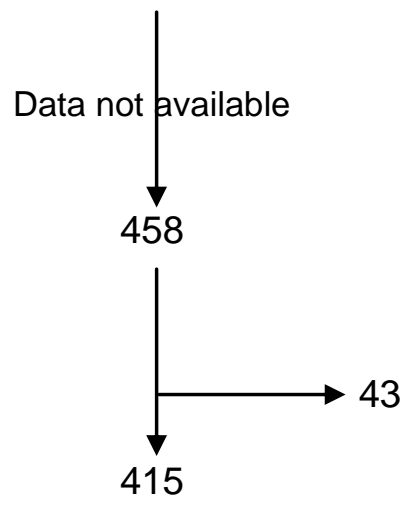

Not applicable

415

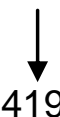

8

9

10 
Figure 2: Tuberculosis case notifications and episodes of culture positive M. tuberculosis with drug susceptibility testing (DST) results available for at least rifampicin and isoniazid, per 100000 per year, 2002 to 2008, companies A (left) and B (right). Table shows numbers of cases notified for each year, per company, and number (percentage) culture positive M. tuberculosis with DST.

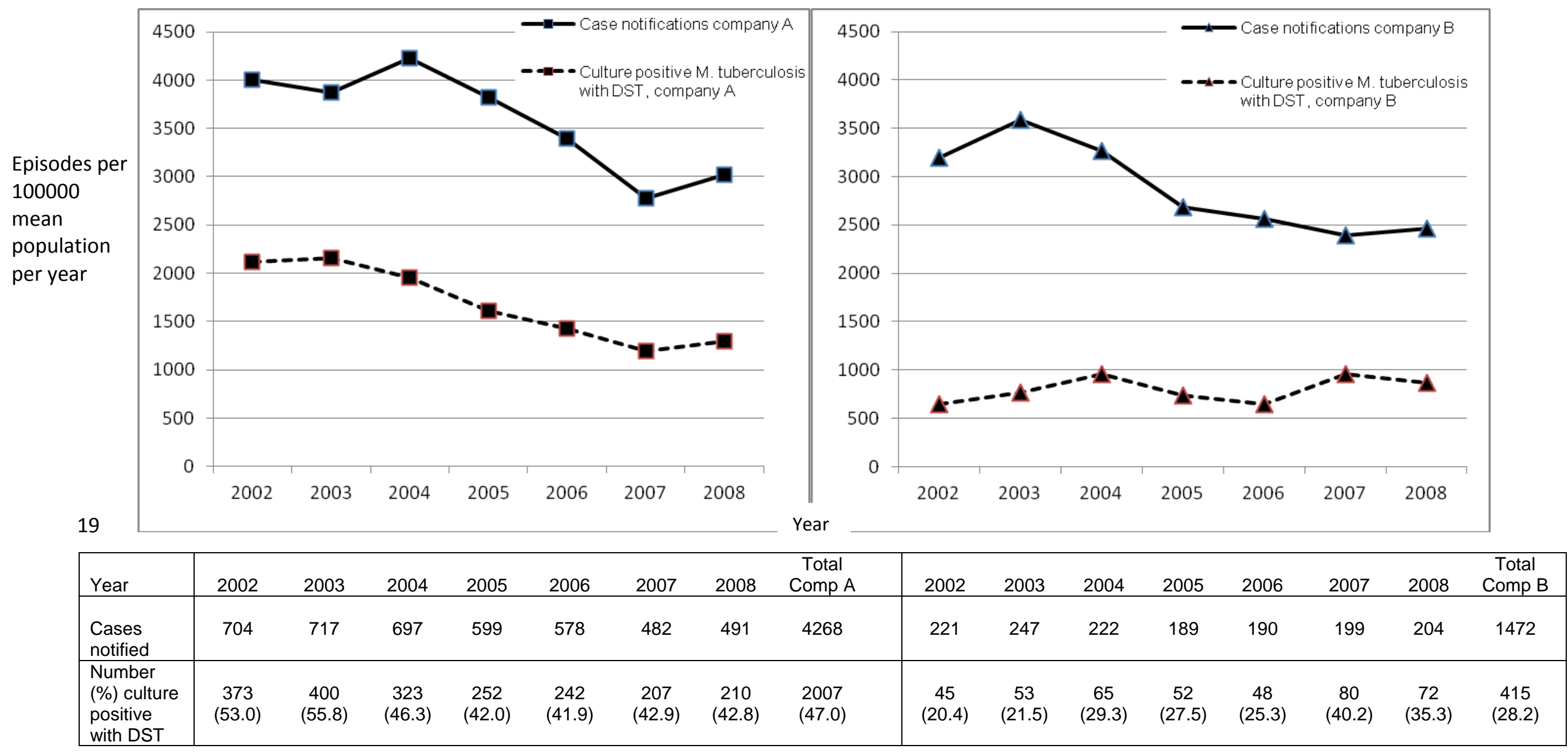

20 
21 Figure 3: Case notification rates for drug-resistant tuberculosis 2002-8: companies A (left) and B (right) Table shows numbers of episodes per 22100,000 mean population as plotted

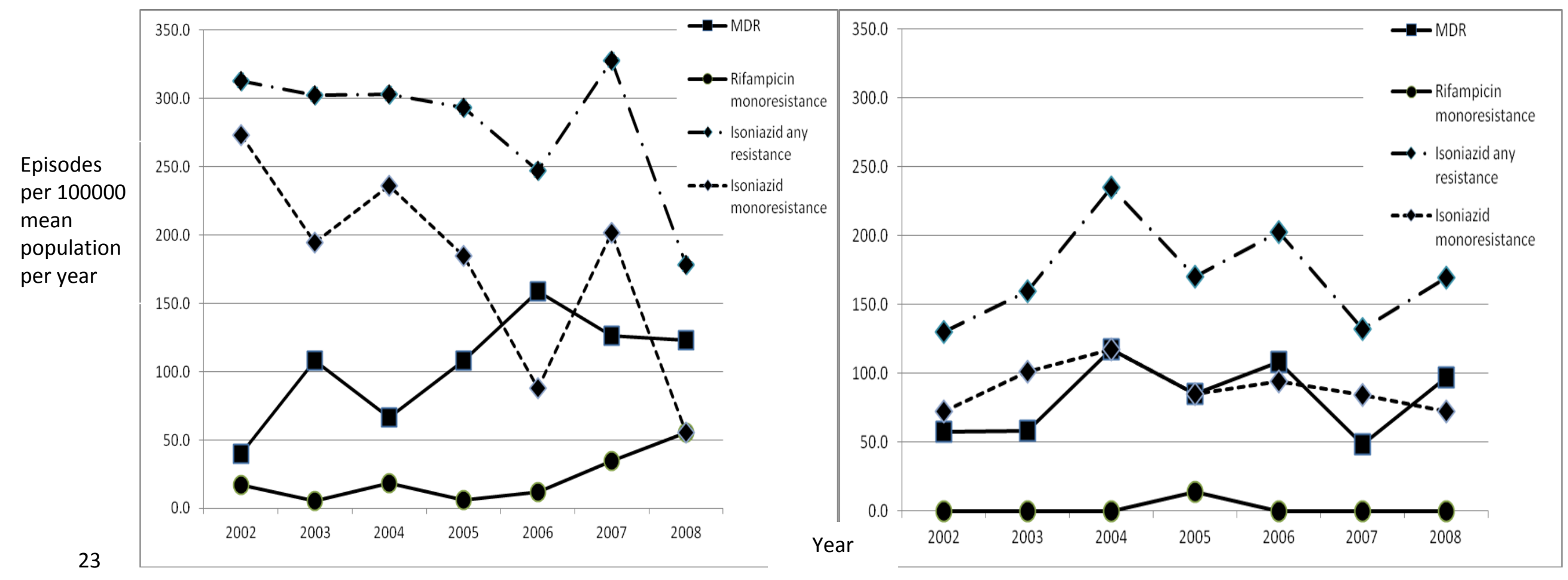

\begin{tabular}{|c|c|c|c|c|c|c|c|c|c|c|c|c|c|c|}
\hline $\begin{array}{l}\text { Number of } \\
\text { episodes }\end{array}$ & 2002 & 2003 & 2004 & 2005 & 2006 & 2007 & 2008 & 2002 & 2003 & 2004 & 2005 & 2006 & 2007 & 2008 \\
\hline MDR & 7 & 20 & 11 & 17 & 27 & 22 & 20 & 4 & 4 & 8 & 6 & 8 & 4 & 8 \\
\hline Rif monores. & 3 & 1 & 3 & 1 & 2 & 6 & 9 & 0 & 0 & 0 & 1 & 0 & 0 & 0 \\
\hline INH monores. & 48 & 36 & 39 & 29 & 15 & 35 & 9 & 5 & 7 & 8 & 6 & 7 & 7 & 6 \\
\hline
\end{tabular}


Figure 4: Drug resistance as a percentage of all M. tuberculosis culture positive episodes among previously untreated cases, 2002-8: companies A (left) and B (right)

27

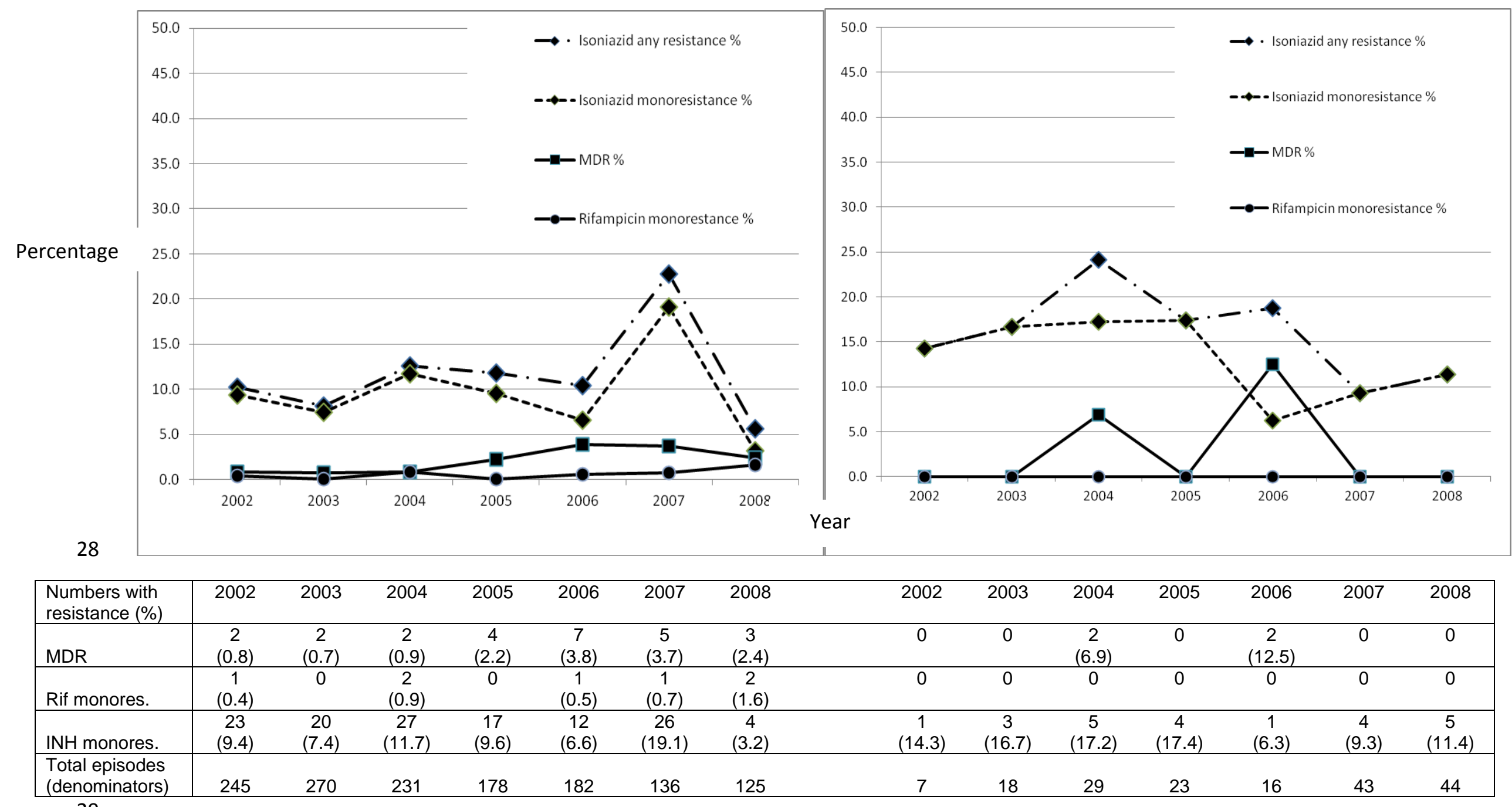
29 
30 Figure 5: Drug resistance as a percentage of all M. tuberculosis culture positive episodes among previously treated cases, 2002-8: companies 31 A (left) and B (right)

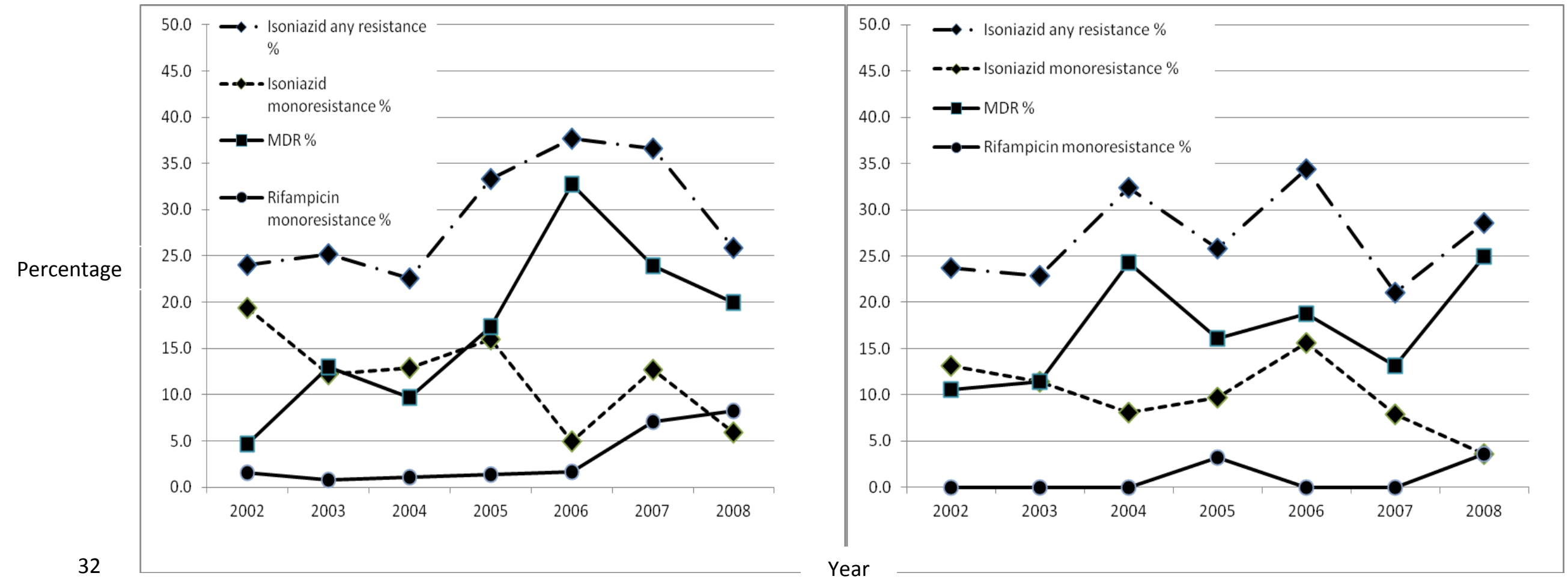

\begin{tabular}{|c|c|c|c|c|c|c|c|c|c|c|c|c|c|c|}
\hline $\begin{array}{l}\text { Percentage with } \\
\text { resistance }\end{array}$ & 2002 & 2003 & 2004 & 2005 & 2006 & 2007 & 2008 & 2002 & 2003 & 2004 & 2005 & 2006 & 2007 & 2008 \\
\hline MDR & $\begin{array}{c}6 \\
(4.7)\end{array}$ & $\begin{array}{c}17 \\
(13.0)\end{array}$ & $\begin{array}{c}9 \\
(9.7)\end{array}$ & $\begin{array}{c}13 \\
(17.3)\end{array}$ & $\begin{array}{c}20 \\
(32.8)\end{array}$ & $\begin{array}{c}17 \\
(23.9)\end{array}$ & $\begin{array}{c}17 \\
(20.0)\end{array}$ & $\begin{array}{c}4 \\
(10.4)\end{array}$ & $\begin{array}{c}4 \\
(11.4)\end{array}$ & $\begin{array}{c}9 \\
(24.3)\end{array}$ & $\begin{array}{c}5 \\
(16.1)\end{array}$ & $\begin{array}{c}6 \\
(18.8)\end{array}$ & $\begin{array}{c}5 \\
(13.2)\end{array}$ & $\begin{array}{c}7 \\
(25.0)\end{array}$ \\
\hline Rif monores. & $\begin{array}{c}2 \\
(1.6)\end{array}$ & $\begin{array}{c}1 \\
(0.8) \\
\end{array}$ & $\begin{array}{c}1 \\
(1.1) \\
\end{array}$ & $\begin{array}{c}1 \\
(1.3)\end{array}$ & $\begin{array}{c}1 \\
(1.6)\end{array}$ & $\begin{array}{c}5 \\
(7.0) \\
\end{array}$ & $\begin{array}{c}7 \\
(8.2) \\
\end{array}$ & 0 & 0 & 0 & $\begin{array}{c}1 \\
(3.2) \\
\end{array}$ & 0 & 0 & $\begin{array}{c}1 \\
(3.6) \\
\end{array}$ \\
\hline INH monores. & $\begin{array}{c}25 \\
(19.4) \\
\end{array}$ & $\begin{array}{c}16 \\
(12.2)\end{array}$ & $\begin{array}{c}12 \\
(12.9)\end{array}$ & $\begin{array}{c}12 \\
(16.0)\end{array}$ & $\begin{array}{c}3 \\
(4.9) \\
\end{array}$ & $\begin{array}{c}9 \\
(12.7) \\
\end{array}$ & $\begin{array}{c}5 \\
(5.9) \\
\end{array}$ & $\begin{array}{c}5 \\
(13.2) \\
\end{array}$ & $\begin{array}{c}4 \\
(11.4) \\
\end{array}$ & $\begin{array}{c}3 \\
(8.1) \\
\end{array}$ & $\begin{array}{c}3 \\
(9.7) \\
\end{array}$ & $\begin{array}{c}5 \\
(15.6)\end{array}$ & $\begin{array}{c}3 \\
(7.9) \\
\end{array}$ & $\begin{array}{c}1 \\
(3.6) \\
\end{array}$ \\
\hline $\begin{array}{l}\text { Total episodes } \\
\text { (denominators) }\end{array}$ & 129 & 131 & 93 & 75 & 61 & 71 & 85 & 38 & 35 & 37 & 31 & 32 & 38 & 28 \\
\hline
\end{tabular}


